\title{
http://bjas.journals.ekb.eg \\ Posterior Vitreous Detachment after Phacoemulsification with and without Anterior Chamber Maintainer
}

AA.El Shayeb, T.T.Soliman, M.A.Heikal and I.A.El-Saadani

Ophthalmology Dept., Faculty of Medicine, Benha Univ.,Benha, Egypt

E-Mail: ibrahimabdelkhalek28@gmail.com

\begin{abstract}
The effect of anterior chamber maintainer on the incidence of posterior vitreous detachment after phacoemulsification. In the current study, our work is to inspect incidence of occurrence of PVD after one M and 3 Ms after uneventful phacoemulsification with and without ACM , In patients with complete vitreal attachment preoperatively. The current study, revealed that the development of postoperative PVD after phacoemulsification at 1 month in $9.7 \%$ in group A and 15.2\% in group B, While at 3 months PVD developed in $12.9 \%$ (8 eyes of 62 eyes) in group A, and $27.2 \%$ ( 25 eyes of 92 eyes) in group B. the incidence of PVD among group A between non- high myopic \& high myopic patients, there were no significant difference at 1 month 5.6\% and $15.4 \%$ and at 3 months were $8.3 \%$ and $19.2 \%$ in non-high myopic and high myopic patients respectively But in group B the incidence of PVD at $1 \mathrm{M}$ and $3 \mathrm{Ms}$ was significantly higher among high myopic patients than non-high myopia, at one month was $6.2 \%, 37.0 \%$ and at three months $13.8 \%, 59.3 \%$ in non-high myopia and high myopic patients respectively. Conclusions: The current study suggest that improving anterior chamber stability by using ACM especially in high myope patients and younger age minimize incidence of PVD after phacoemulsification surgery.
\end{abstract}

Keywords: Anterior Chamber Maintainer, Posterior Vitreous Detachment, Phacoemulsification.

\section{Introduction}

Phacoemulsification surgery lead to changes in vitreous due to the loss of stabilization from the crysetalline lens [1]. Vitreous alterations occurring after phaco in eyes without preoperative PVD may be involved in the occurrence of pseudophakic RD later on [2].The most common reason of rhegmatogenous $\mathrm{RD}$ is thought to be PVD due to retinal traction [3].Surgical trauma should be considered a risk element for PVD. Anterior chamber breakdown is a important factor for change of the vitreoretinal interface. Improving AC stability may prevent destabilization of the vitreous attachments and may lead to reduction in the occurrence of PVD by using a ACM and continued irrigation during the whole phacoemulsification surgery avoiding momentary collapse of $\mathrm{AC}$ when handpiece removed from the eye [4]. The aim of this study is to assess the effect of anterior chamber maintainer on incidence of posterior vitreous detachment after phacoemulsification.

\section{Material and methods}

This is a prospective non-randomized comparative interventional study.A 174 eyes of 169 patients with cataract undergoing phacoemulsification at Ophthalmology department of Benha University Hospital between May 2018 and December 2019 were engaged after informed written consent was acquired from all patients before involved in the study.

\subsection{Inclusion criteria}

All patients who have cataract with complete vitroretinal attachment who underwent uneventful phacoemulsification surgery.

\subsection{Exclusion criteria}

Patients with preoperative partial or complete PVD ,Patients with history of glaucoma or uveitis and Patients with previous eye surgery.

Patients with Intraoperative complications e.g., capsular tear and vitreous loss, (20 eyes of 15 patients) and patients missing established follow-up visits were excluded from this study.

Cases (154 eyes of 154 patients) were separated into 2 groups at the baseline based on the technique: Group. A (62 eyes ):Phacoemulsification with an Anterior Chamber Maintainer (ACM).Group_B (92 eyes ):Phacoemulsification without an ACM.

All patients were subjected to: Ultrasound biometry, high myope was defined with axial length 26 $\mathrm{mm}$ or more .B-mode ultrasonography, PVD was diagnosed by separation of the posterior vitreous from the inner retina on U/S , the detached vitreous seen on $\mathrm{U} / \mathrm{S}$ as a low-reflective membrane floating in the vitreous cavity in front of the retina .

\subsection{Surgical procedure}

Group A: Once emulsification is complete, the phaco tip remains inside eye with opened irrigation .Before removing the phaco handpiece from the eye, the second bottle of Ringer Lactate connected to ACM is opened, and irrigation through $\mathrm{ACM}$ is introduced . Cortex aspiration is then performed by bimanual I/A through side ports, the eye inflated under continuous irrigation from ACM throughout I/A and during changing hands . Before IOL implantation, we fill the capsular bag and anterior chamber with OVD under continuous irrigation with reducing gradually the flow inside the AC by lowering the bottle height connected to ACM .After IOL implantation, the aspiration of the viscoelastic was done with open flow of the ACM. All incisions was closed by injecting ringer lactate in the corneal stroma and the removal of the ACM was done by simultaneous injection of Ringer Lactate through a 
side- port to maintain $\mathrm{AC}$ formed and prevent $\mathrm{AC}$ collapse.

Group B :We will use the same technique as group A without use of ACM after completing phaco procedure and after irrigation aspiration.

\subsection{Follow up}

Patients were instructed about the symptoms of PVD and retinal tears including sudden onset of flashes, floaters, blurred vision or acurtain encroaching over the peripheral field of vision. They were advised to return immediately for ophthalmic examination if such symptoms arise, irrespective of the scheduled follow up dates._All patients in both groups will be examained 1 day, 3days, 1 week, 1 month and 3 months after surgery.All patients in both groups will be examained by B-scan 1 month and 3 months after surgery to detect PVD.

\section{Results}

Regarding incidence of occurrence of PVD group A and group B , There was no significance in difference between the 2 groups regarding the incidence of PVD at $1 \mathrm{~m}$, while at 3 months, there is significant difference as incidence of PVD was higher at group B Table (1).

Among non-high myopia patients, there was no significance in difference between the 2 groups regarding the incidence of PVD at 1 month or 3 months Table (2).

Among high myopia patients there was no significance in difference between 2 groups regarding the incidence of PVD at 1 month while, its incidence was significantly higher in group B at 3 months Table (3).

Table ( 1) Difference between 2 groups regarding incidence of PVD.

\begin{tabular}{lcccc}
\hline PVD & Group A eyes $(\mathbf{n}=62)$ (No. $(\%)$ & Group B eyes $(\mathbf{n}=92)($ No. $(\%)$ & $\square^{2}$ & P value \\
\hline PVD 1m & & & & \\
No & $56(90.3)$ & $78(84.8)$ & & 0.316 \\
Yes & $6(9.7)$ & $14(15.2)$ & \\
PVD 3m & $54(87.1)$ & $67(72.8)$ & $\square^{2}=4.48$ & 0.034 \\
No & $8(12.9)$ & $25(27.2)$ & & \\
Yes & & & \\
\hline
\end{tabular}

\section{$\square^{2}=$ chi squared test}

Table (2) difference between 2 groups regarding incidence of PVD among non- high myopia patients.

\begin{tabular}{lcccc}
\hline PVD & Group A eyes(n=36) & Group B eyes $(\mathbf{n = 6 5})$ & Fisher's exact test & P value \\
\hline PVD 1 m & & & & \\
No & $34(94.4)$ & $61(93.8)$ & 0.01 & 1.00 \\
Yes & $2(5.6)$ & $4(6.2)$ & & \\
PVD 3 m & $33(91.7)$ & $56(86.2)$ & 0.67 & 0.530 \\
No & $3(8.3)$ & $9(13.8)$ & & \\
Yes & & & & \\
\hline
\end{tabular}

Table (3) Comparison between group A and group B regarding incidence of PVD among high myopia patients .

\begin{tabular}{lcccc}
\hline PVD & $\begin{array}{c}\text { Group A eyes }(\mathrm{n}=26) \\
\text { No. }(\%)\end{array}$ & $\begin{array}{c}\text { Group B eyes }(\mathrm{n}=27) \\
\text { No. }(\%)\end{array}$ & $\square^{2}$ & P value \\
\hline PVD 1 m & & $17(63.0)$ & 3.19 & 0.074 \\
No & $22(84.6)$ & $10(37.0)$ & & \\
Yes & $4(15.4)$ & & & \\
PVD 3 m & & $11(40.7)$ & 8.87 & $\mathbf{0 . 0 0 3}$ \\
No & $21(80.8)$ & $16(59.3)$ & & \\
Yes & $5(19.2)$ & & & \\
\hline
\end{tabular}

\section{Discussion}

Cataract surgery is an independent risk factor for $\mathrm{RD}$ due to postoperative structural alterations in the vitreous. Anatomically, following the removal of the lens, there are changes in the vitreous volume. Add to this , certain biochemical modifications including changes in proteome, viscosity, and macromolecules in the vitreous body were started and may lead to PVD, a known risk factor for Reghmatonous retinal detachment [5].

In the current study, we search for the occurrence of PVD after $1 \mathrm{M}$ and $3 \mathrm{Ms}$ after uneventful phaco cataract surgery with and without ACM , In patients 
with comlete posterior vitreal attachment preoperatively

Our study, revealed that the development of postoperative PVD after phaco cataract surgery at 1 month in $9.7 \%$ (6 eyes) in group A and $15.2 \%$ (14 eyes) in group B, While at 3 months PVD developed in $12.9 \%$ ( 8 eyes of 62 eyes) in group A, and $27.2 \%$ (25 eyes of 92 eyes) in group B. There was no significance in difference between the 2 groups regarding the incidence of PVD at $1 \mathrm{~m}$, while at 3 months, there is significant difference as incidence of PVD was higher at group B.. (Table 1)

Mirashahi found that $20 \%$ of eyes developed PVD 1 week after uncomplicated phaco, $31 \%$ had developed the condition 1 month after, this percentage is higher than we found in our study[6].

Ripandelli reported a PVD incidence of $75.88 \%$ (in $107 / 141$ eyes) in emmetropic eyes without preoperative PVD after 26 months follow up, this percentage is higher than we found in our study [7].

Hilford reported that PVD was detected in 50.8\% of eyes underwent phacoemulsification as compared to $20.8 \%$ in the non-operated fellow eyes, , this percentage is higher than we found in our study [8].

Degremenci evaluated the effect of uneventful phacoemulsification on vitroretinal interface using Bscan and OCT and found that PVD occurs commonly soon after cataract surgery ,PVD developed in $56.66 \%$ at one month and $70 \%$ at three months and $73.33 \%$ at six months .High incidence of PVD because they use OCT and B-scan in evaluating vitroretinal interface [9].

OCT facilitate the detection of early changes of the vitreoretinal interface. Consequently, using supplementary OCT with U/S might lead to detection of a higher number of PVD cases [10].

OCT detects minor changes in the inner retinal surface and posterior vitreous cortex and even stage 1 PVD could easily be detected with OCT [9].

Ivastinovic evaluate the onset of PVD in eyes after uneventful phaco cataract surgery by assessment of vitreoretinal interface changes using both OCT and ultrasound and reported that PVD was detected in (59.2\%) 1 month after surgery and in (71.4\%) 3 months after surgery [10].

The reasons for the difference between the studies are not clear, however, the age, refractive errors, long period of follow up, use of different techniques of phacoemulsification and use of different techniques may explain this difference.

In this study, all patients in both groups were divided into 2 subgroups based on AXL $\geq 26.0 \mathrm{~mm}$, as high myopic eyes is also an established risk factor for occurrence of PVD[11].

In this study, regarding the incidence of PVD among non-high myopia patients, the difference between group A and group B was no significant at one month and three months at 1 month $5.6 \%$ in group $A$ and $6.2 \%$ in group B eyes ,and at 3 months $8.3 \%$ in group $A$ eyes and $13.8 \%$ in group B eyes Table (2).
While the incidence of PVD among high myopia patients, the difference between group A and group B at 1 month was non-significant, while the incidence of PVD was significantly higher in group B at $\mathrm{t} 3$ months, At 1 month $15.4 \%$ in group A eyes and $37.0 \%$ in group B eye ,At 3 months $19.2 \%$ in group A eyes and $59.3 \%$ in group B eyes Table ( 3 ).

The incidence of PVD was significantly higher among high myopia patients in group B at three months 59.3\%

The explanations for high PVD incidence after phacoemulsification in group B eyes (without use of anterior chamber maintainer) are not clear.

Mirshahi propose that forward movements of the anterior hyaloid, which happen after phaco cataract surgery may be the responsible for initiation of cascade of PVD process [6].

Sallet suggest that Surgical trauma should be considered a risk element for intiation of PVD. Anterior chamber breakdown is a important factor for changes occur at the level of the vitreoretinal interface [4].

So despite different studies on incidence of initiation of post vitreous detachment after phacoemulsification there is no till now prevention measures to prevent or to delay post vitreous detachment.

But we suggest that use of anterior chamber maintainer to keep anterior chamber inflated throgouh out phaco surgery may decrease incidence of PVD after phaco especially in high myope.

\section{References}

[1] S. J. Haug and R. B. Bhisitkul, "Risk factors of retinal detachments after phaco cataract surgery.", current opinion in ophthalmology 23.1, PP. 7-11, 2012.

[2] M.A.Coppé, and G.Lapucci,"posterior vitreous detachment and Retinal Detachment after cataract surgery ." ,current opinions in ophthalmology 19.3 , PP. 239-242, 2008.

[3] G.Nassrallah , M.Kondoff, M.Ross, \& J.Deschenes, PVD and incidence of delayed retinal break: a retrospective, 2-years study in an academic centre." Canadian J., ophthalmology 54.4, PP. 509-512, 2019.

[4] G.Sallet " Improving eye anterior chamber stability during ". Crsteurope, 2011.

[5] K.Kassem, Y.Greenwald, A.Achiron, I.Hech ,\& A.Bukelman " occurence of Retinal detachments after cataract surgery:." J., ophthalmology , 2018.

[6] A.Mirshahi, F. Hoehn, K.Lorenz, K.kohen, \& A.Hattenbach. "Incidence of posterior vitreous detachment after cataract ." J., cataract \& refractive surgery 35.6 , PP. 987-991, 2009.

[7]G.Ripandelli,A.Coppé, V.Pairisi, D.Oelzi, C.Scaassa, A.Chiaravalloti \& M. Stirpe", post vitreous detachment and RD following cataract surgery." ophthalmology 114.4, PP. 692-697, 2007.

[8]D. Hilford, M.Hilford, , A.Mathew, \&P.J. Polkinghorne, "PVD following cataract surgery." eye 23.6 , P.1388, 2009.

[9] C.Degirmenci . ,F.Afrashi, J.Mentes, Z.Oztas ,S.Nalcaci ,C.Akkin "Evaluation of PVD after uneventful 
phacoemulsification surgery by optical coherence tomography and US." clinical and experimental optometry 100.1, PP. 49-53, 2017.

[10] I.Domagoj, C.Schwab, A.Borkenstein, E. Lackner, A. Wedrich, and M.V.Pare "Evolution of early changes at the vitrOretinal interface after cataract surgery detected by OCT and ultrasonography." American J., ophthalmology 153.4 , PP.705-709, 2012.

[11] T.Hikichi"Time course of development of post vitreous detachment after phacoemulsification surgery." ophthalmology 119.10 , PP.2102-2107, 2012. 